\title{
Toward an Intelligent High Frequency AC Distributed Power System: Part II; Analytical Modelling and Experimental Realization
}

\author{
Patrick C. K. Luk ${ }^{1}$, Tareq S. El-Hasan ${ }^{2}$
}

\begin{abstract}
High frequency AC (HFAC) distributed power systems (DPS), where electric power is delivered at up to multi-kHz via cables, is an alternative means to conventional centralized power systems. This paper explores the means by which real-time information can be achieved without installing additional physical communication channels on an existing $50 \mathrm{kHz}$ current-fed HFAC DPS in lighting applications. A communication protocol is methodologically developed to facilitate robust and efficient inter-device real-time communication. In addition, analytical modelling for the current fed coupling circuit is presented. The utility of an intelligent HFAC DPS is demonstrated by experimental results from comprehensive circuit level implementation.
\end{abstract}

Keywords: Modems, data communication, intelligent power distribution, energy management, power distribution systems, high frequency AC, lighting systems.

\section{Nomenclature:}

$C_{p} \quad$ Parallel capacitance

$C_{s} \quad$ Series capacitance

$|H(s)| \quad$ Magnitude of the voltage transfer function

$k \quad$ Tuning ratio

$k_{p} \quad$ Peak tuning ratio

$L_{p} \quad$ Parallel inductance

$L_{s} \quad$ Series inductance

$n \quad$ Number of receiving node

$N \quad$ Number of transmission modem

$|P(s)| \quad$ Magnitude of the ratio of $|H(s)|$ to the current

$Q_{p m} \quad$ Quality factor of the parallel branches

$Q_{s m} \quad$ Quality factor of the series branches

$R_{I N} \quad$ Input impedance of the modem at the carrier frequency

$R_{T} \quad$ Total impedance of the remaining modems

$V \quad$ Transmit amplifier output voltage

$Z_{\text {INVERTER }}$ Inverter impedance

$\omega_{p m} \quad$ Resonant frequency of parallel branches

$\omega_{s m} \quad$ Resonant frequency of series branches

\section{Introduction}

Transmitting and receiving data over the traditional Distribution Power System (DPS) using Power Line Communication (PLC) or Broadband over Power Line (BPL) have been in service for a while [1] - [3]. However, such systems were not designed for frequencies other than $50 / 60 \mathrm{~Hz}$ and they tend to have limitation on data transfer speed and capacity. Alternative solution was first introduced by NASA in 1980s to power the on-board equipment in space station using High Frequency AC (HFAC) [4]. Later, other research activities but limited in scope of HFAC system have been reported in [5] - [9]. Results revealed that such system offers numerous potential benefits including but not limited to the higher speed of data transfer, flexibility of voltage levels, significant savings in number of system components and improving system dynamic response. However, progress of work on HFAC DPS system in our daily life has been very slow. Some initial work on HFAC lighting was reported in [10], [11]. Works on intelligent distributed lighting systems have been introduced, but they were based on conventional power using wireless sensors [12], or separate communication networks [13]. Other research activities on smart control of lighting involving new power grids have been tackled in [14], [15]. Marginal work has been focused on developing converters for lighting applications [16] - [18]. 
In a typical HFAC network, it is anticipated that there would be many agents all communicating with each other over the same HFAC bus, causing potential data collision. Bus arbitration mechanism is necessary to ensure that collisions are avoided and detected if they do happen. As the communication is required mainly for telemetry purposes, the required data bitrate is relatively low. A data rate of less than $1 \mathrm{kbps}$ is sufficient for most applications [19], [20].

In this paper a HFAC data modem is proposed to facilitate communication over the HFAC power bus without the need for additional communication channel. Implemented on a lighting application, the analytical model of the coupling circuit is developed, buss access mechanism is defied and the realization of an intelligent HFAC distributed power network is demonstrated by the cost-effectiveness and a high degree of retrofitability offered by the proposed data modem.

\section{Analytical Modelling Of The Coupling Circuit}

Before proceeding with the analytical modelling, one has to consider the requirements of the current-fed coupling circuits and the communication challenges associated with current fed system [21] - [23]. In a current-fed system, the modems are effectively connected in series to the HFAC current loop. Fig. 1 shows a simplified equivalent circuit for a number of modems connected to a HFAC current loop. In this case it is assumed that no loads are present in the system. To ensure that the impedance of the inverter ( $Z_{\text {INVERTER }}$ ) does not attenuate the communication signal, a tuned band-pass filter can be added in parallel with ( $Z_{\text {INVERTER }}$ ) to offer an alternate low resistance path for the communication signal. The simplified equivalent modem circuit is shown in Fig. 2. The constraints stated above can be achieved by using a coupling circuit of the form shown in Fig. 3. Note that the series and parallel branch orientation is crucial for proper operation of the circuit. Both the series and parallel branch are tuned to the carrier frequency. The parallel branch offers a low impedance path for the bus current.

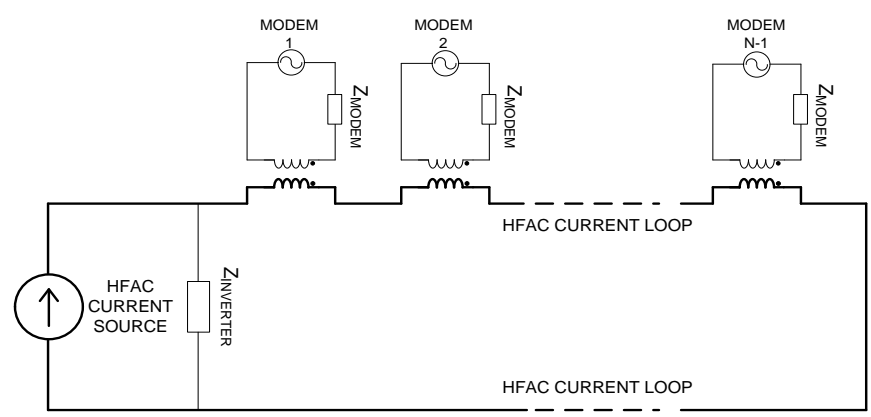

Fig. 1. Multiple modems on a current-fed HFAC bus.

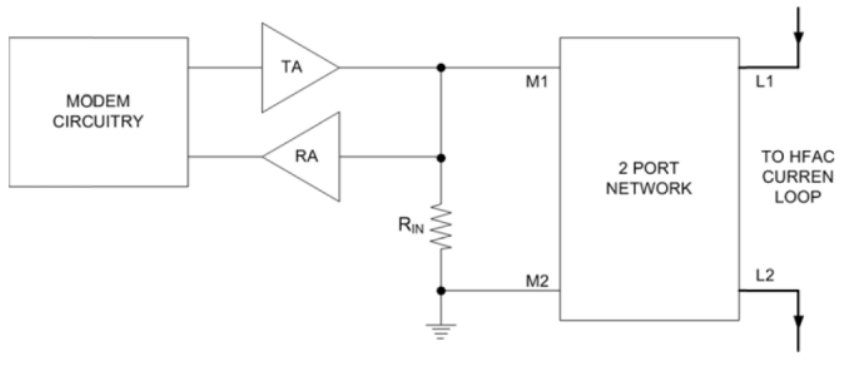

Fig. 2. Simplified representation of the modem line interface stage for a current-fed system.

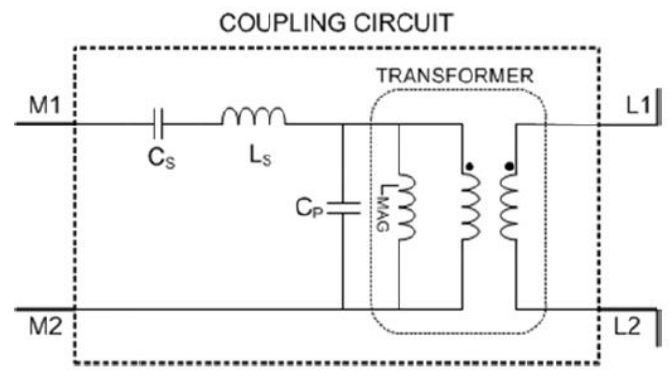

Fig. 3. Modem coupling circuit for a current-fed system.

Assuming the resonant elements in the above circuits are tightly tuned, the input impedance of the modem at the carrier frequency is simply equal to the value of $R_{I N}$. The impedance seen by the transmitting modem in a system with modem can be represented as in Fig. 4. If the transmit amplifier output voltage is $V$, then the voltage across each receiving node is equal to $V /(n-1)$. Therefore even under ideal conditions with no line and load impedance, amplitude of the received signal falls as the number of modems increase.

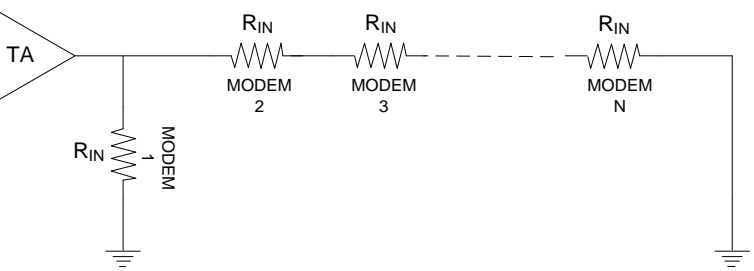

Fig. 4. Transmit mode equivalent impedance model.

When two modems are simultaneously transmitting as presented in Fig. 5, a signal of amplitude $V$ due to selftransmission will be present at the input node of MODEM 1. In addition, another voltage component due to the simultaneous transmission of modem $\mathrm{N}$ will appear across the input node of MODEM 1. The amplitude of this component will be $V /(N-1)$. Therefore the signal seen at the input of both modems will consist of the sum of both the self-transmission component and the simultaneous transmission by the neighbouring modem. If the number of modems in the system is large, the amplitude of the voltage due to the transmission of the 
neighbouring modem will be small and may be totally overwhelmed by the self-transmission component. This may potentially impair the collision detection capability. Any load connected to the loop increases the total resistance and further aggravates these problems. Therefore a tuned band-pass bypass filter should always be used across all loads to offer an alternate low resistance path for the communication signals.

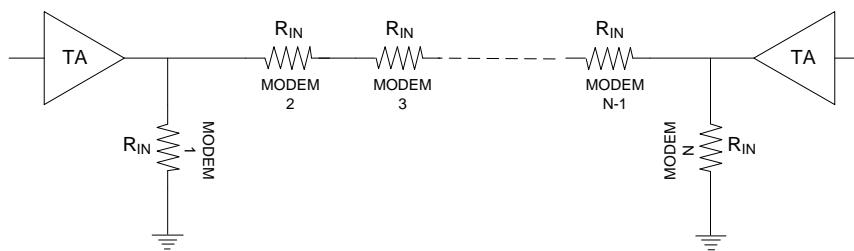

Fig. 5. Multiple transmit mode equivalent impedance model.

In transmit mode, the equivalent circuit of the modem and the coupling circuit is shown in Fig. 6 where $R_{T}$ is total impedance of the remaining modems in the system and is given by

$$
R_{T}=(N-1) R_{I N}
$$

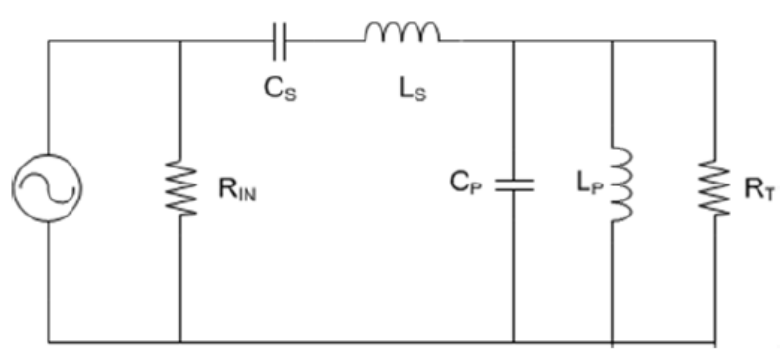

Fig. 6. Coupling interface equivalent circuit- transmit mode.

The quality factor and the resonant frequency of the series $Q_{s m}$ and parallel $Q_{p m}$ branches are defined as

$$
\begin{aligned}
& Q_{s m}=\frac{\sqrt{L_{S}}}{R_{I N} \sqrt{C_{S}}} \\
& Q_{p m}=\frac{R_{I N} \sqrt{C_{P}}}{\sqrt{L_{P}}} \\
& \omega_{s m}=\frac{1}{\sqrt{L_{S} C_{S}}} \\
& \omega_{p m}=\frac{1}{\sqrt{L_{P} C_{P}}}
\end{aligned}
$$

Both the series and parallel branches are tuned to the same frequency. The tuning ratio is defined as the ratio of the drive frequency to the resonant frequency and is given as

$$
k=\frac{\omega_{o}}{\omega_{s m}}=\frac{\omega_{o}}{\omega_{p m}}
$$

Using the definitions 2-5, the magnitude of the voltage transfer function can be expressed as

$|H(s)|=\frac{k^{2}(N-1)}{\sqrt{\left(k^{3}-k\right)^{2} Q_{s m}^{2}+\left(k^{2}-\left(k^{2}-1\right)^{2} Q_{s m}^{2} Q_{p m}^{2}\right)^{2}(N-1)^{2}}}$

In receive mode, the modem is driven by a current source and can be represented as in Fig. 7. The transfer function of the voltage across $R_{I N}$ to the current is given as

$$
|P(s)|=\frac{k^{2}}{\sqrt{\left(k^{3}-k^{2}\right)^{2} Q_{p m}^{2}+\left(k^{2}-\left(k^{2}-1\right)^{2} Q_{s m} Q_{p m}\right)^{2}}}
$$

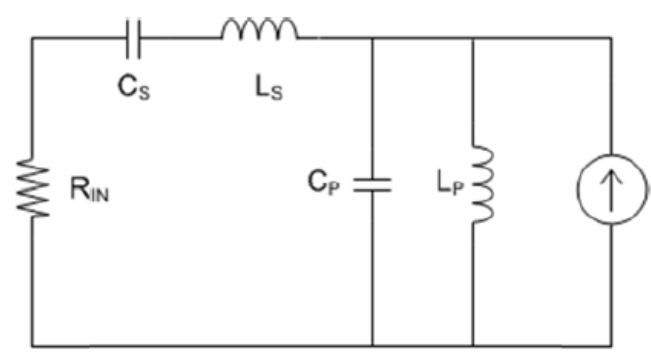

Fig. 7. Coupling interface equivalent circuit- receive mode

Equations 7 and 8 can be used to identify the constraints imposed on the selection the coupling circuit element. At this stage it is interesting to note that the magnitude of $P(s)$ is independent of the number of modems while the magnitude of $H(s)$ is not. The output of the modem is well controlled and predictable. However in receive mode, the content of the current source is less predictable. It represents the sum of the communication current, the bus current and any noise that may have been picked up by the bus. Therefore the coupling circuit will be designed with the desired response of $P(s)$ taking precedence over $H(s)$.

The first constrain is defined to limit the magnitude of $P(s)$ below a certain frequency to be smaller than a prescribed value $\varepsilon$. This is to ensure that the HFAC bus current is sufficiently attenuated. This can be mathematically represented by 9 . It is recommended that $k_{o}$ be selected to represent at least the $10^{\text {th }}$ harmonic of the bus current. Substituting $k=0.045$ into 9 and 8 , the first constraint imposed on the coupling circuit is derived and is given in (10). 


$$
|P(s)|_{k \leq k_{o}}<\varepsilon
$$

$$
\sqrt{\frac{1}{491.829 Q_{p m}^{2}+\left(1-491.829 Q_{p m} Q_{s m}\right)^{2}}} \leq \varepsilon
$$

The plot of the magnitude of $H(s)$ for various values of $Q_{s m}$ is shown in Fig. 8. It can be seen that the magnitude plot appears to have a peak for some values of $Q_{s m}$. The frequency at which these peaks occur and the magnitude of the peaks depend on the values of $Q_{s m}$, $Q_{p m}$ and $N$.

Fig. 9 shows how the magnitude of $H(s)$ varies as the number of modems in the system change for fixed values of $Q_{s m}$ and $Q_{p m}$. It can be observed from the figure that the frequency at which the peak occurs remains fairly constant but the peak amplitude increases rapidly with $N$.

Taking the derivative of 7 with respect to $k$ and equating it to zero, the value of $k$ at which the peaks occur can be calculated. This is given in (11).

$$
k_{p}=\sqrt{\frac{\left(J_{a}+J_{b}\right) \pm \sqrt{\left(J_{a}+J_{b}\right)^{2}-J_{b}^{2}}}{J_{b}}}
$$

where

$$
\begin{gathered}
J_{a}=2(N-1)^{2} Q_{p m}-Q_{s m} \\
J_{b}=4(N-1)^{2} Q_{p m}^{2} Q_{s m}
\end{gathered}
$$

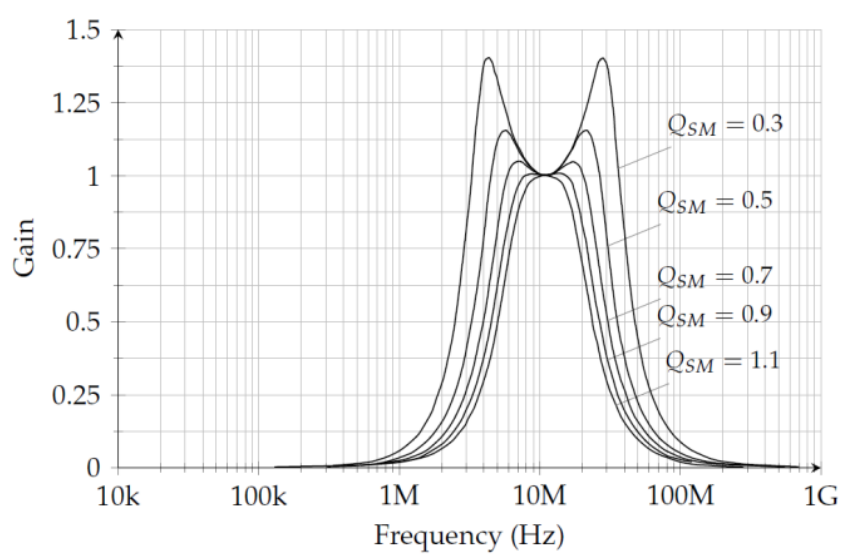

Fig. 8. Frequency response of $H(s)$ for various $Q_{s m}$.

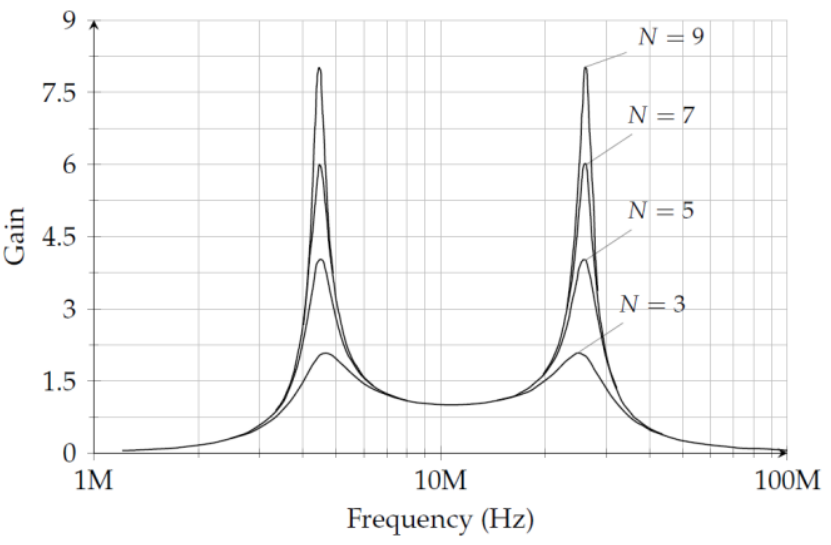

Fig. 9 Frequency response of $\mathrm{H}(\mathrm{s})$ for various number of modems.

The magnitude of the peaks can be found by substituting 11 into 7 . It can be further shown from 11 that the peaks in $|H(s)|$ start to appear when condition given in 14 is met. Similar peaking behaviour can be observed in the magnitude of $P(s)$ when condition given in 15 is met.

$$
\begin{gathered}
Q_{s m}<2(N-1)^{2} Q_{p m} \\
Q_{s m}>\frac{Q_{p m}}{2}
\end{gathered}
$$

The relationship between the frequencies at which the peaks occur to the number of modems in the system has interesting properties. This plot is shown in Fig. 10 for various values of $Q_{s m}$ and $Q_{p m}$. It can be seen that for values of $N$ greater than 4 , the values of $k_{p}$ remains constant. However the magnitude of the peaks increase as more modems are present in the system. A good approximation for $k_{p}$ can be found by letting $N \rightarrow \infty$ in 11 and solving the resulting equation yields the following relation between $k_{p}$ and the quality factors:

$$
Q_{s m} Q_{p m} \approx \frac{k_{p}^{2}}{\left(k_{p}^{2}-1\right)^{2}}
$$

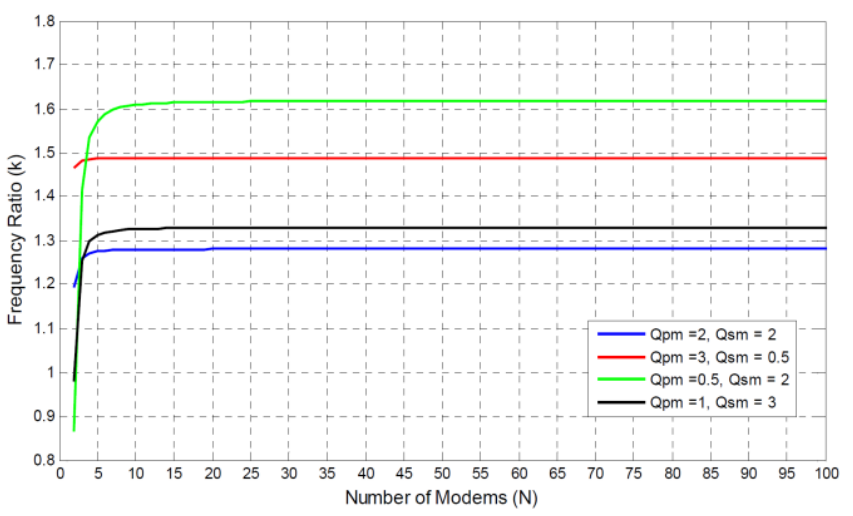

Fig. 10: $k_{p}$ as a function of the number of modems. 
The magnitude of the peaks grows rapidly with the increase of the number of modems in the system as can be seen from Fig. 11. Therefore it is important to ensure that the output of the transmit amplifier does not have any harmonic frequency component at these frequencies. Although the transmit low-pass filter is designed to have a steep roll-off rate, it is preferable to ensure that the peaks occur at even harmonics of the carrier frequency. The reason for this is that the output of the transmit VCO is a symmetrical square wave and does not contain even harmonics.

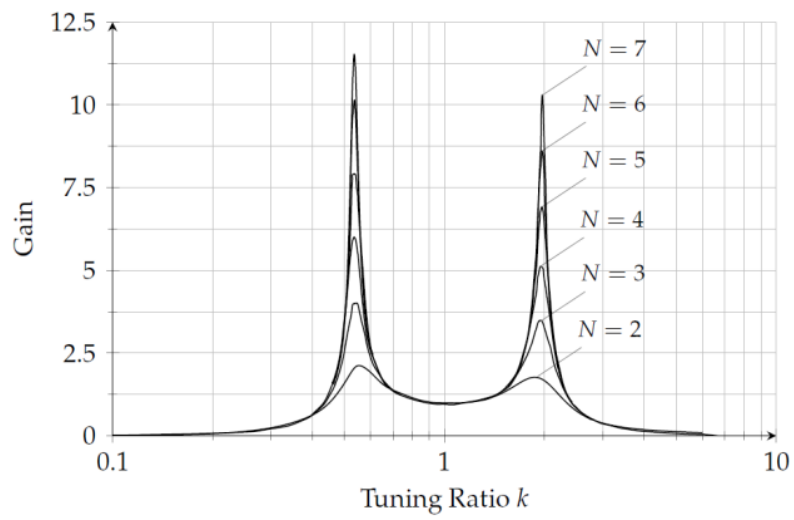

Fig. 11. Frequency response of $H(s)$ for the coupling circuit design.

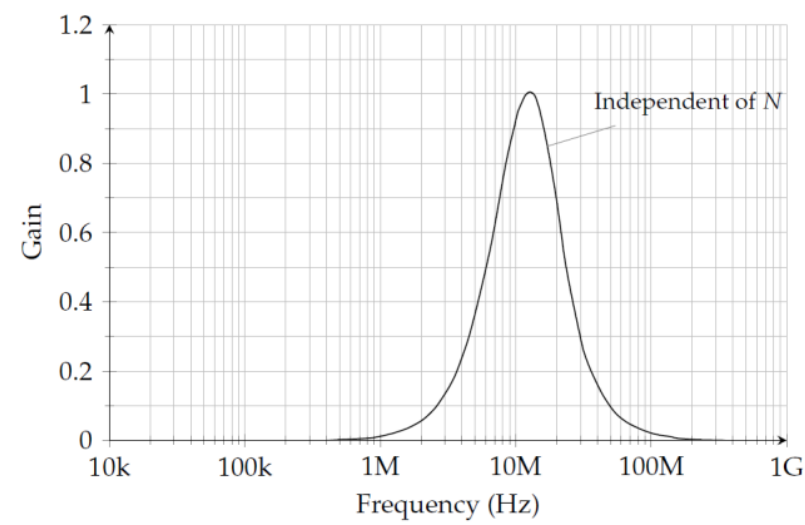

Fig. 12. Frequency response of $P(s)$ for the coupling circuit design.

In summary, equations 10,15 and 16 can be used as a guide to select ideal values for the coupling circuit. Table I shows a set of computed values for the first 6 even harmonics. Firstly for each even harmonic component the product of $Q_{s m} Q_{p m}$ is computed using 16. Then the minimum value of $Q_{p m}$ is determined using 10 for a desired value of $\varepsilon$. In this case, a value of 0.05 was used. It is proposed that a headroom of at least 20 is added to the computed $Q_{p m}$ value and furthermore this must be checked to ensure compliance with 15 . Once the value of $Q_{p m}$ is finalized, then $Q_{s m}$ can be determined using 16 , and the physical values of the coupling circuit can then be determined. In general it is desired that the coupling circuit be designed to ensure the peaks of $H(s)$ occur at the second harmonics. This would give then best performance, however in some cases this may impose high constraints on the values of the components required for the coupling circuit. The first row in Table I gives the values of the coupling circuit elements that ensure the peak response of $H(s)$ occur at the second harmonic. These values are proposed for this design. The simulated response of $H(s)$ and $P(s)$ for $L_{s}=560$, $C_{s}=390, L_{p}=1$ and $C_{p}=180$ are shown in Fig. 11 and Fig. 12 respectively. It can be seen that the simulation matches the expected response based on the approximate modelling.

\section{Frame Format and Bus Access Mechanism}

\section{III.1. Frame Format}

A frame or data packet is constructed by appending other relevant information to the raw data such as addressing information, error detection and synchronization features. The proposed frame format for this application is shown in Fig. 13.

TABLE I

Calculation of Coupling Circuit Element Values

\begin{tabular}{|c|c|c|c|c|c|c|c|c|}
\hline $\mathrm{kp}$ & $\begin{array}{c}\text { Qpm x } \\
\text { Qsm }\end{array}$ & $\begin{array}{l}\text { Min } \\
\text { Qpm }\end{array}$ & $\begin{array}{c}\text { Qpm } \\
+10 \% \\
\end{array}$ & Qsm & Ls & Cs & Lp & $\mathrm{Cp}$ \\
\hline 2 & 0.444 & 0.943 & 1.131 & 0.393 & $5.68 \mathrm{E}-07$ & $3.68 \mathrm{E}-10$ & $1.28 \mathrm{E}-06$ & $1.64 \mathrm{E}-10$ \\
\hline 4 & 0.071 & 0.377 & 0.453 & 0.157 & 2.27E-07 & $9.21 \mathrm{E}-10$ & 3.20E-06 & $6.55 \mathrm{E}-11$ \\
\hline 6 & 0.029 & 0.670 & 0.804 & 0.037 & $5.29 \mathrm{E}-08$ & $3.96 \mathrm{E}-09$ & $1.80 \mathrm{E}-06$ & $1.16 \mathrm{E}-10$ \\
\hline 8 & 0.016 & 0.850 & 1.020 & 0.016 & $2.29 \mathrm{E}-08$ & $9.15 \mathrm{E}-09$ & $1.42 \mathrm{E}-06$ & $1.48 \mathrm{E}-10$ \\
\hline 10 & 0.010 & 0.890 & 1.068 & 0.010 & $1.38 \mathrm{E}-08$ & $1.51 \mathrm{E}-08$ & $1.35 \mathrm{E}-06$ & $1.55 \mathrm{E}-10$ \\
\hline 12 & 0.007 & 0.900 & 1.080 & 0.007 & $9.43 \mathrm{E}-09$ & $2.22 \mathrm{E}-08$ & $1.34 \mathrm{E}-06$ & $1.56 \mathrm{E}-10$ \\
\hline
\end{tabular}




\begin{tabular}{|c|c|c|c|c|c|c|c|c|}
\hline PREAMBLE & $\begin{array}{c}\text { START } \\
\text { FRAME } \\
\text { DELIMITER }\end{array}$ & $\begin{array}{c}\text { DEST. } \\
\text { ADDRESS }\end{array}$ & $\begin{array}{c}\text { SOURCE } \\
\text { ADDRESS }\end{array}$ & $\begin{array}{c}\text { DATA } \\
\text { LENGTH } \\
\text { FIELD }\end{array}$ & $\begin{array}{c}\text { TYPE } \\
\text { FIELD }\end{array}$ & DATA & $\begin{array}{c}\text { CHECK } \\
\text { SUM }\end{array}$ & $\begin{array}{c}\text { END } \\
\text { FRAME } \\
\text { DELIMITER }\end{array}$ \\
\hline 3 BYTES
\end{tabular}

This format is a simplified implementation derived from the frame structure of the Ethernet protocol (IEEE 802.3). The total frame length is 18 bytes. The first 3 bytes are the preamble, defined as 0xAA. These are followed by a start frame delimiter (SFD) which is 1 byte in length and is defined as OxAB. The SFD is used to indicate the start of the frame. Next, a 1 byte destination address (DA) and source address (SA) is used for packet routing and addressing. The data length field (DLF) is used to indicate the number of bytes of payload data present. The data type field (DTF) is used to indicate the type of frame; this could be used to distinguish control frames from data frames if necessary. Next, eight bytes of actual raw data is sent followed by an eight bit checksum (CS) information. Finally an end frame delimiter (EFD) is used to indicate the end of the current frame. The EFD is defined as $0 \mathrm{xF} 0$.

\section{III.2. Bus Access Mechanism}

In this design, carrier sense multiple access with collision detection (CSMA/CD) is implemented. Before a node attempts to transmit information over the HFAC bus, the medium is first checked to ensure it is idle. This is done by reading the carrier sense signal. If the medium is not idle, then the modem waits until it becomes available. When the bus is idle, the modem starts to transmit the frame.

It is possible that two or more modems start transmitting at the same instant. Therefore as the modems are transmitting, it is programmed to monitor its own transmission to ensure no data collision has occurred. Collision detection is implemented by comparing the received information with the transmitted frame. If a mismatch is detected, it is assumed that a data collision has occurred. The received information is discarded and the frame transmission is aborted immediately. Then the transmitter sends a jam signal to ensure all nodes in the system are made aware of the collision. The jam signal consists of a string of alternating 1's and 0's with a length of 4 bytes. After the end of the jam signal transmission, all modems wishing to transmit will wait for a random period of time before attempting a retransmission. The backoff time is typical chosen to be small compared to the frame duration. However if there are many modems in the system and high communication traffic is likely, then the probability of collision will be higher under these conditions. Therefore the backoff time will need to be determined relative to the number of detected collision.

\section{Experimental Setup and Results}

The experimental setup to test the capability of the system is shown in Fig. 14 and Fig. 15. A constant current HFAC inverter were used to establish a loop current of 1.35 RMS at 50. A 12 LED load was connected to the loop via a DC voltage rectification module (VRM). Two modems were connected within the loop and data was transmitted over the HFAC current bus. Two bypass stubs consisting of a series LC circuit tuned to the carrier frequency was used across the inverter and the VRM to provide a low impedance path to the communication signal. The raw transmit data was generated from an external host, a PC in this experiment, and based on this information the transmit frame was constructed. The NRZ clock frequency is approximately 38.983 , and one bit of data is transmitted at every clock period.

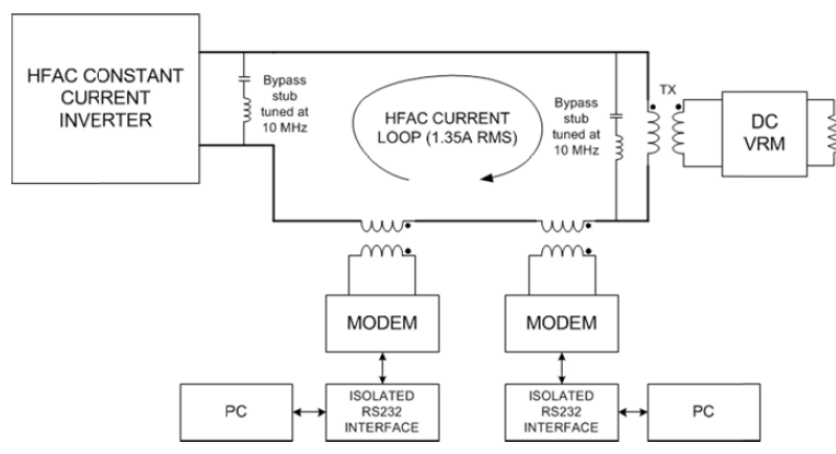

Fig. 14. Experimental setup of communication over HFAC current-fed bus.

In Fig. 16 the transmitted NRZ data, the clock signal of the transmitter, the encoded Manchester data stream and the loop current waveform are shown. The reconstructed received signal at the receiver modem, representing the output of the pulse shaping block, is shown. It can be observed that the amplitude of the logic ' 1 ' carrier is approximately 15 and the logic ' 0 ' carrier amplitude is approximately twice that of the logic ' 1 ' carrier amplitude. It is important to note that although the loop current waveform resembles an amplitude modulated signal, it is in fact a FSK modulated signal with the logic '1' carrier deliberately attenuated. This is done to model the non idealities in the filter frequency response and the attenuation of the signal due to the inductance of the HFAC bus which could result in the logic ' 1 ' carrier frequency (12.5) to be attenuated by a greater degree as shown. 
Figure 17 shows that the receiver is able to reconstruct the correct logic levels despite the variation in the amplitude of the carrier signal. Using the same setup, the communication features were tested with the bus power turned ON (1.35 RMS at 50). Fig. 18 shows the waveform of the transmitted information captured at the source modem and the received waveform captured at the destination modem. It can be seen that the information was successfully transmitted over a powered HFAC bus. The bus current is 1.35 RMS with a frequency of 50.24. A stable and consistent bitrate of $38 \mathrm{kbps}$ was achieved.

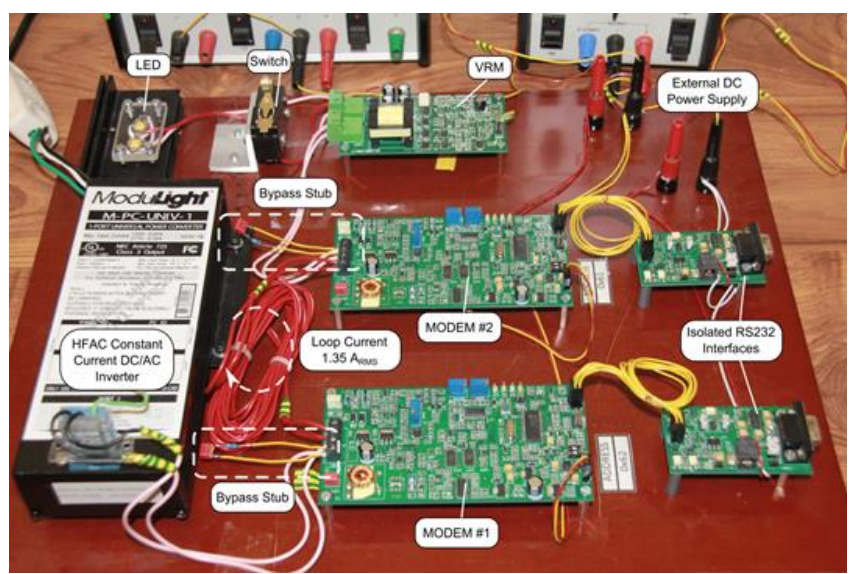

Fig. 15. HFAC modem experimental setup.

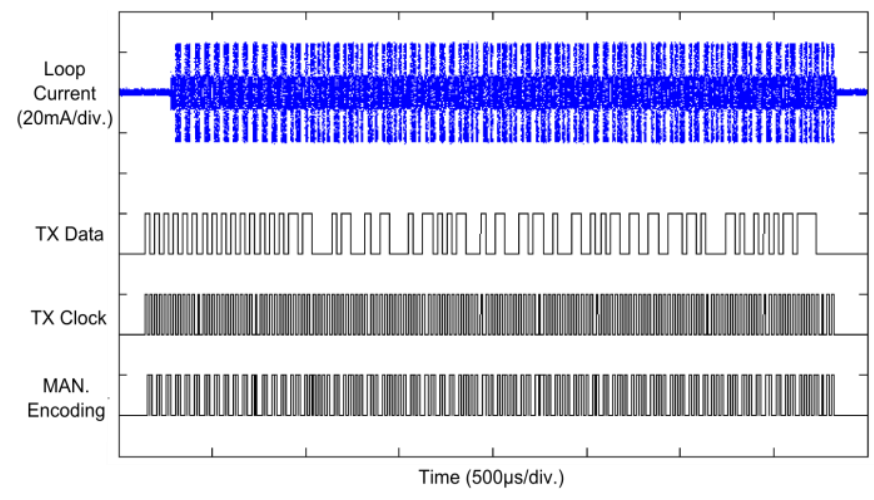

Fig. 16. a) Communication signals and loop current waveforms; Complete transmission frame.

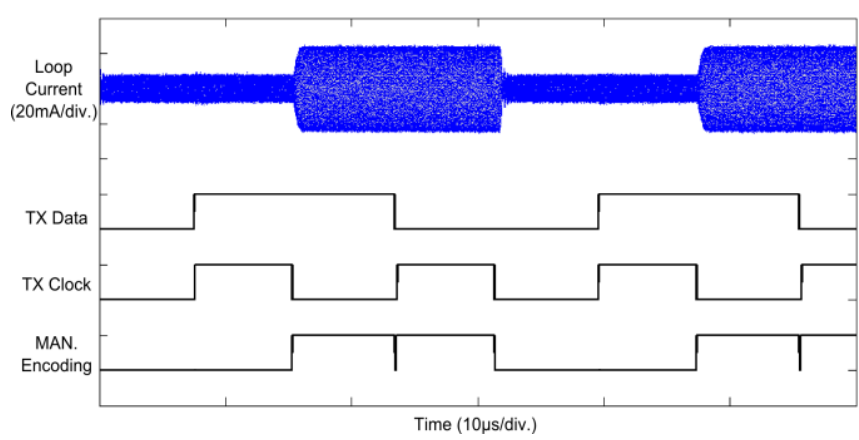

Fig. 16. b) Communication signals and loop current waveforms; Zoomed in image showing individual communication bits.

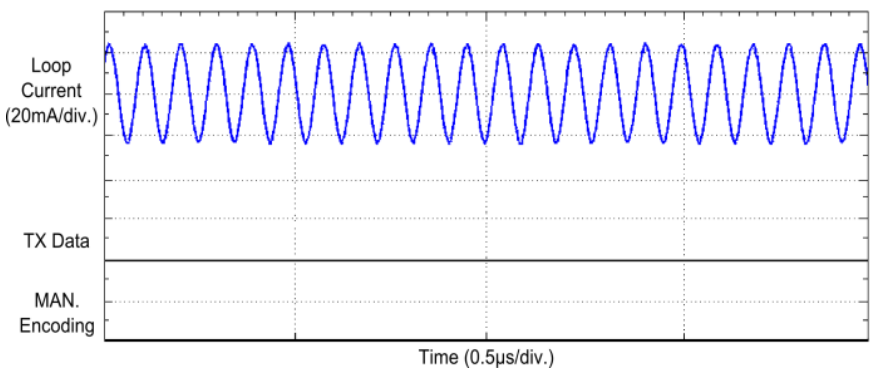

(a) logic ' 0 '

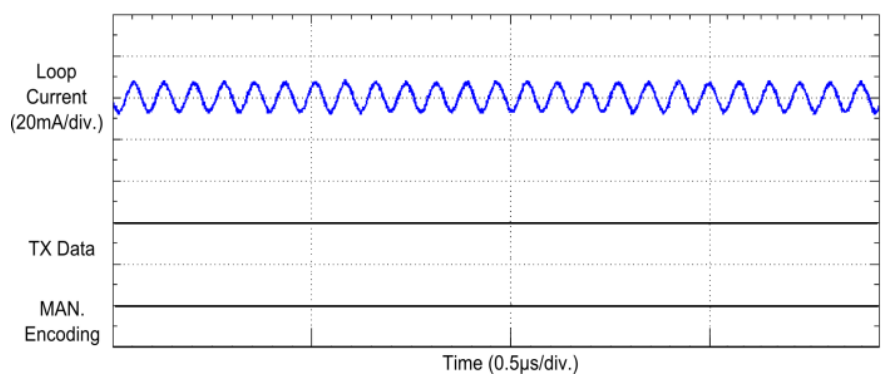

(b) $\operatorname{logic}$ ' 1 '

Fig. 17. Reconstructed signals at the receiver

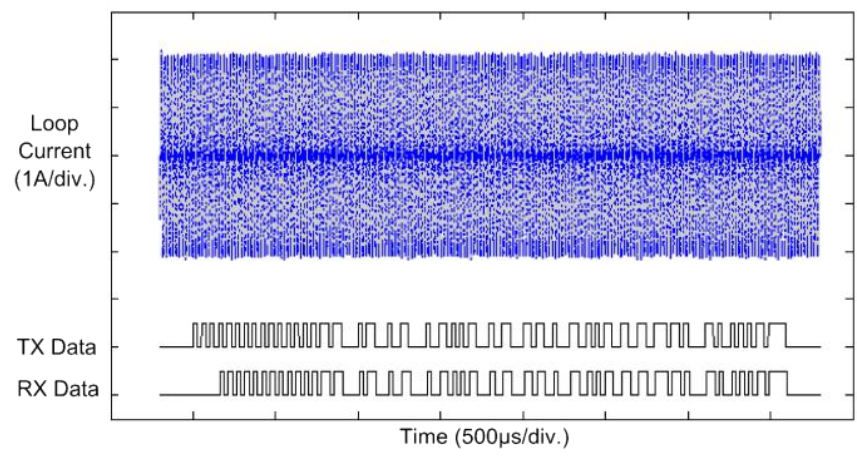

Fig. 18. Transmitted and received NRZ data over a powered HFAC bus.

\section{Conclusion}

This paper proposed analytical modelling of the current fed coupling circuit and experimental reaslisation of HFAC distributed power system. Specifically, a data modem capable of implementing bidirectional communication at a data rate of $38 \mathrm{kbps}$ over a $50 \mathrm{kHz}$ HFAC current-fed power bus in a lighting application is successfully demonstrated. Mathematical models were derived and used as a guide to select ideal values for the coupling circuit. Simulation results were obtained based on the derived models and they were close to the expected response.

The paper also presented the relationship between the frequencies at which the peaks of the frequency response occur to the number of modems in the system. It was also shown that the magnitude of the peaks grows rapidly with the increase of the number of modems in the system. Therefore it is important to ensure that the output of the 
transmit amplifier does not have any harmonic frequency component at the tuned frequencies.

A carrier sense multi access with collision (CSMACD) detection bus arbitration scheme was implemented to enable multiple node addressable bidirectional communication, a crucial provision for devices to communicate reliably and seamlessly on the power bus.

Experimentations of the proposed modem showed that the information was successfully transmitted over a powered HFAC bus. A stable and consistent bitrate of 38 kbps was achieved. The proposed enabling communication framework presents significant potentials that can be exploited in implementing intelligent power management schemes for HFAC distributed power systems and other multiple inverter and multiple load HFAC power networks.

\section{Acknowledgement}

The authors would like to thank Steven Lourdes for his contribution in the experimental validation and the Technology Strategy Board of the United Kingdom for supporting the High Frequency Power and Distribution with Data project (TP/5/POW/6/I/H0049D) in collaboration with Juice Technology Ltd, as well as the financial provision by Zarqa University in Jordan for the publication of the article.

\section{References}

[1] Cataliotti A., Di Cara D., Fiorelli R., Tine G., "Power-Line Communication in Medium-Voltage System: Simulation Model and Onfield Experimental Tests," IEEE Transactions on Power Delivery, Vol. 27, No. 1, pp. 62 - 69, 2012.

[2] Della Giustina D., Ferrari P., Flammini A., Rinaldi S., Sisinni E., "Automation of Distribution Grids With IEC 61850: A First Approach Using Broadband Power Line Communication," IEEE Transactions on Instrumentation and Measurement, Vol. 62, No., pp. $2372-23839,2013$.

[3] El Haj Y., Albasha L., El-Hag A., Mir H., "Data communication through distribution networks for smart grid applications," IET on Science, Measurement \& Technology, Vo. 9, No. 6, pp. $774-781,2015$.

[4] D. Renz, R. C. Finke, N. J. Stevens, J. E. Triner, and I. G. Hansen, "Design considerations for large space electric power systems," NASA Technical Memorandum 83064, 1983.

[5] P. Sood and T. Lipo, "Power conversion distribution system using a high-frequency AC link," IEEE Trans. Ind. Appl., vol. 24, no. 2, pp. 288-300, Mar./Apr. 1988.

[6] P. Jain and H. Pinheiro, "Hybrid high frequency AC power distribution architecture for telecommunication systems," IEEE Trans. Aerosp. Electron. Syst., vol. 35, no. 1, pp. 138-147, Jan. 1999.

[7] S. Luo and I. Batarseh, "A review of distributed power systems. Part II. high frequency AC distributed power systems," IEEE Trans. Aerosp. Electron. Syst., vol. 21, no. 6, pp. 5-14, Jun. 2006.

[8] Jain, P. , Pahlevaninezhad, M. , Pan, S. , Drobnik, J. A, "Review of High-Frequency Power Distribution Systems: For Space, Telecommunication, and Computer Applications," Transactions on Power Electronics, Vol. 29, No. 8, pp. $3852-$ 3863, Aug 2014.
[9] Jun Zeng, Junfeng Liu, Jinming Yang, Fei Luo, “A Voltage-Feed High-Frequency Resonant Inverter With Controlled Current Output as a High-Frequency AC Power Source", IEEE Transactions on Power Electronics, Volume: 30, Issue: 9, pp. $4854-4863$, Sep 2015.

[10] C.-L. Kuo, T.-J. Liang, K.-H. Chen, and J.-F. Chen, "Design and implementation of high frequency AC-LED driver with digital dimming," in IEEE Int. Symp. on Circuits and Systems, Jun. 2010, pp. 3713-3716

[11] S.-Y. Ng, P. Luk, and K. Jinupun, "High frequency AC distributed power system for fluorescent lighting," in IEEE 14th European Conf. on Power Electronics and Applications, Aug. 2011, pp. 1-10.

[12] M. Nakamura, A. Sakurai, and J. Nakamura, "Distributed environment control using wireless sensor/actuator networks for lighting applications," Sensors, vol. 9, no. 11, pp. 8593-8609, 2009.

[13] Y. Kasahara, M. Miki, and M. Yoshimi, "Preliminary evaluation of the intelligent lighting system with distributed control modules," in 11th Int. Conf. Intelligent Systems Design and Applications (ISDA), 2011, pp. 283-288.

[14] C. K. Lee, S. Li, and S. Hui, "A design methodology for smart LED lighting systems powered by weakly regulated renewable power grids," IEEE Trans. Smart Grid, vol. 2, no. 3, pp. 548-554, Aug. 2011.

[15] Y. K. Tan, T. P. Huynh, and Z. Wang, "Smart personal sensor network control for energy saving in DC grid powered LED lighting system," IEEE Trans. Smart Grid, vol. 4, no. 2, pp. 669 676, May 2013

[16] M. G. L. Roes, J. Duarte, and M. Hendrix, "Disturbance observerbased control of a dual-output LLC converter for solidstate lighting applications," IEEE Trans. Power Electron., vol. 26, no. 7, pp. 2018-2027, Aug. 2011.

[17] W. Yu, J.-S. Lai, H. Ma, and C. Zheng, "High-efficiency DC-DC converter with twin bus for dimmable LED lighting," IEEE Trans. Power Electron., vol. 26, no. 8, pp. 2095-2100, Aug. 2011.

[18] M. Arias, D. Lamar, F. Linera, D. Balocco, A. Diallo, and J. Sebastian, "Design of a soft-switching asymmetrical half-bridge converter as second stage of an LED driver for street lighting application," IEEE Trans. Power Electron., vol. 27, no. 3, pp. 1608-1621, Feb. 2012.

[19] Kansal P., Bose A., "Bandwidth and Latency Requirements for Smart Transmission Grid Applications," IEEE Transactions on Smart Grid, Vol. 3, No. 3, pp. 1344 - 1352, 2012.

[20] Bumiller G., Lampe L., Hrasnica H., "Power line communication networks for large-scale control and automation systems," IEEE Communications Magazine, Vol. 48, No. 4, pp. $106-113,2010$.

[21] S.Aldhaher, P.C.K.Luk, Khalil El Khamlichi Drissi, J.F.Whidborne,"High Input Voltage High Frequency Class Rectifiers for Resonant Inductive Links,"Power Electronics, IEEE Transactions on, Vol..99, pp.1-9, 2014. DOI: 10.1109/TPEL.2014.2316170.

[22] S. Aldhaher, P.-K. Luk, and J. Whidborne, "Tuning Class E inverters applied in inductive links using saturable reactors," IEEE Trans. Power Electron. vol. 29, no. 6, pp. 2969-2978, Jun. 2014.

[23] P. C. K. Luk, Tareq S. El-Hasan, "Toward an Intelligent High Frequency AC Distributed Power System: Part I; Conceptual Design", International Review of Electrical Engineering (I.R.E.E.), Vol. 11, No. 5, 2016 


\title{
Authors' information
}

\author{
${ }^{1}$ School of Engineering, Cranfield University, Bedford, UK
}

${ }^{2}$ Electrical Engineering Department, Zarqa University, Jordan

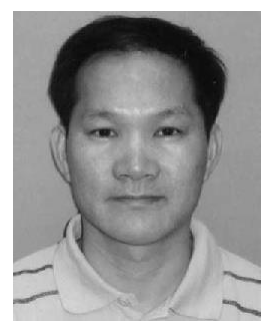

Patrick Chi-Kwong Luk (M'92-SM'08) was born in Hong Kong. He received the High Diploma with merits (BSc) in electrical engineering from Hong Kong Polytechnic University, Hong Kong, in 1983, the M.Phil. degree in electrical engineering from Sheffield University, U.K., in 1989, and the Ph.D. degree in electrical engineering from the University of South Wales in 1992.

He started his career in industry in 1983, first as an Assistant Engineer at GEC (H.K.) and then as an Application Engineer at Polytek Engineering Co. (H.K.). In 1986, he became a Researcher with the Industrial Centre, PolyU. Since 1988, he has held academic positions at the University of South Wales, Robert Gordon University, Aberdeen, U.K., and University of Hertfordshire, U.K. He joined Cranfield University, Shrivenham, U.K., in 2002, where currently he is a Chair Professor in Electrical Engineering and Head of the Electric Power and Drives Group, School of Engineering. Currently he is the Chairman of the IEEE UK\&RI Power Electronics Chapter. He has published over 130 technical papers and co-holder of several patents in power electronics, motor drives, and control. His main current research interests include electrical drives for electric vehicles and renewable energy applications, and high-frequency ac power distributions.

He won the 2011 IET Premium Award in Electric Power Applications.

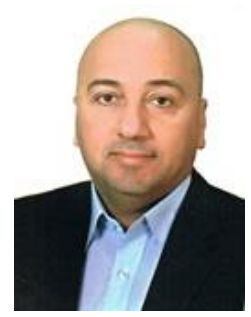

Tareq S. El-Hasan received his BSc degrees in Electrical Engineering from Mu'tah University of Jordan in 1989. Since then he has started his career path at Jordan Armed Forces (JAF) as an electrical engineer then as a senior design engineer until Sep 1999 where he has held the position of chief of building and automation systems. In Oct 1999 he has been awarded a full $\mathrm{PhD}$ scholarship sponsored by JAF. He received his $\mathrm{PhD}$ degree in Electrical Engineering from University of Hertfordshire in the UK in Jan 2003. He has worked for Defense Industry at King Abdullah II Design and Development Bureau (KAADB) in Jordan from March 2003 until March 2010 in which he has held several senior technical and managerial positions. He has joined the electrical engineering department at Zarqa University in Jordan since Sep 2010 until now. He held the position of electrical engineering chair from Sep 2011 until Sep 2013 and the vice dean for college of engineering from Sep 2014 - Sep 2015. His research interests are in High-Speed PM Machines, Electrical Drives and control, Pulsed Power and renewable energy. In addition to his industrial and academic experience, Dr. Tareq has maintained wellestablished records in program and project management during his work in industry for more than 20 years and during his work as a prime consultant for Method Co. in Kingdom of Saudi Arabia. He has successfully managed multidisciplinary projects and has successfully led the implementation of project/program management office (PMO) at the enterprise level in several sectors. He has published several scientific papers in International Journals and Conference proceedings. 